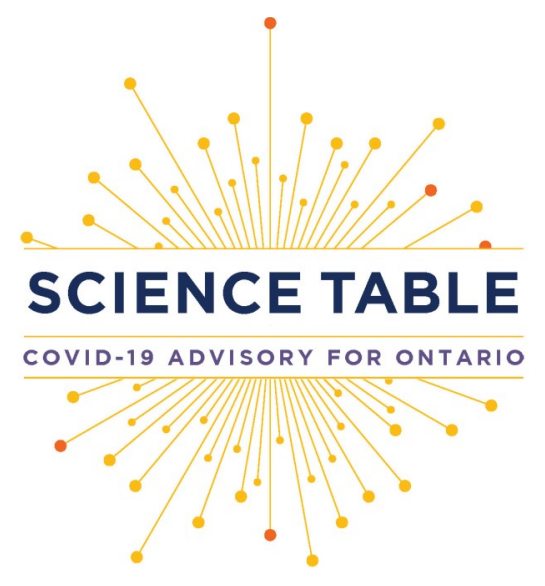

Version 1.0

Published: January 21, 2021

Citation: Stall NM, McGeer A, Maltsev A, et al. The Impact of the Speed of Vaccine Rollout on COVID-19 Cases and Deaths in Ontario Long-Term Care Homes. Science Briefs of the Ontario COVID-19 Science Advisory Table. 2021;2(8). https:// doi.org/10.47326/ocsat.2021.02.08.1.0

Author Affiliations: The affiliations of the members of the Ontario COVID-19 Science Advisory Table can be found at https:// covid19-sciencetable.ca/.

Declarations of Interest: The declarations of interest of the members of the Ontario COVID-19 Science Advisory Table can be found at https://covid19-sciencetable.ca/.

About Us: The Ontario COVID-19 Science Advisory Table is a group of scientific experts and health system leaders who evaluate and report on emerging evidence relevant to the COVID-19 pandemic, to inform Ontario's response. Our mandate is to provide weekly summaries of relevant scientific evidence for the COVID-19 Health Command Table of the Province of Ontario, integrating information from existing scientific tables, Ontario's universities and agencies, and the best global evidence. The science Table summarizes its findings for the Health Command Table and the public in Science Briefs.

Correspondence to: Secretariat of the Ontario COVID-19 Science Advisory Table (info@covid19-sciencetable.ca)

Copyright: 2020 Ontario COVID-19 Science Advisory Table. This is an open access document distributed under the terms of the Creative Commons Attribution License, which permits unrestricted use, distribution, and reproduction in any medium, provided that the original work is properly cited.

The views and findings expressed in this Science Brief are those of the authors and do not necessarily reflect the views of all of the members of the Ontario COVID-19 Science Advisory Table and its partners.

\title{
The Impact of the Speed of Vaccine Rollout on COVID-19 Cases and Deaths in Ontario Long-Term Care Homes
}

Nathan M. Stall, Allison McGeer, Antonina Maltsev, Isaac I. Bogoch, Kevin A. Brown, Gerald A. Evans, Fahad Razak, Beate Sander, Brian Schwartz, Tania Watts, Peter Jüni on behalf of the Ontario COVID-19 Science Advisory Table

\section{Key Message}

Accelerating the rollout of Ontario's COVID-19 vaccine such that all LTC residents receive the first dose of a COVID-19 vaccine by January 31, 2021 would prevent a projected 600 COVID-19 cases and 115 deaths by March 31, 2021 when compared with the province's current plan to vaccinate all LTC residents by February 15, 2021. Projections indicate that further acceleration of the rollout would prevent even more COVID-19 cases and deaths.

If vaccine supply is limited, the early provision of first doses of a COVID-19 vaccine to LTC home residents is likely to be more beneficial than the on-schedule provision of second doses to health care workers outside of LTC homes. All LTC residents should receive the second dose according to approved vaccination schedules.

\section{Background}

Ontario LTC home residents have experienced disproportionately high rates of SARSCoV-2 infection and COVID-19 mortality. As of January 17, 2021, there has been a total of 13,337 cumulative SARS-CoV-2 infections and 3,212 cumulative COVID-19 deaths among LTC residents, accounting for $59.1 \%$ of Ontario's total 5,433 COVID-19 deaths. ${ }^{1}$

Ontario's COVID-19 vaccine rollout began on December 14, 2020, with the Province identifying its approximately 70,000 LTC home residents as a priority group to receive the initial supply of COVID-19 vaccines. ${ }^{2}$

Recently, Ontario's COVID-19 vaccination program specified January 21,2021 , as the target date for the provision of first doses of the vaccine to LTC residents in the Toronto, Peel, York and Windsor-Essex public health units. ${ }^{3}$ As of January 17, 2021, these regions accounted for 160 of 623 of Ontario's LTC homes (25.7\%), 25,703 of 78,764 of Ontario's licensed LTC beds (32.6\%), 21,854 of 69,799 of Ontario's occupied LTC beds (31.3\%), and 97 of 248 of Ontario's active COVID-19 LTC home outbreaks (39.1\%).

The province's current plan is to provide the first dose of a COVID-19 vaccine to all LTC residents by February 15, 2021. As of January 20, 2021, end of day, 39,098 of 69,799 LTC residents have received the first dose of a COVID-19 vaccine (56.0\%).

\section{Questions}

How many COVID-19 cases and deaths in LTC residents could be prevented by March 31, 2021 if the first dose of the COVID-19 vaccine were provided to all Ontario LTC residents by January 21 or January 31, as compared with the currently 


\section{Findings}

A total of 12,200 COVID-19 cases among LTC residents are projected between January 13, and March 31, 2021, without vaccination (best case 2200; worst case, 22,000 ). Figure 1 presents the projected number of prevented COVID-19 cases according to the three vaccine rollout scenarios.

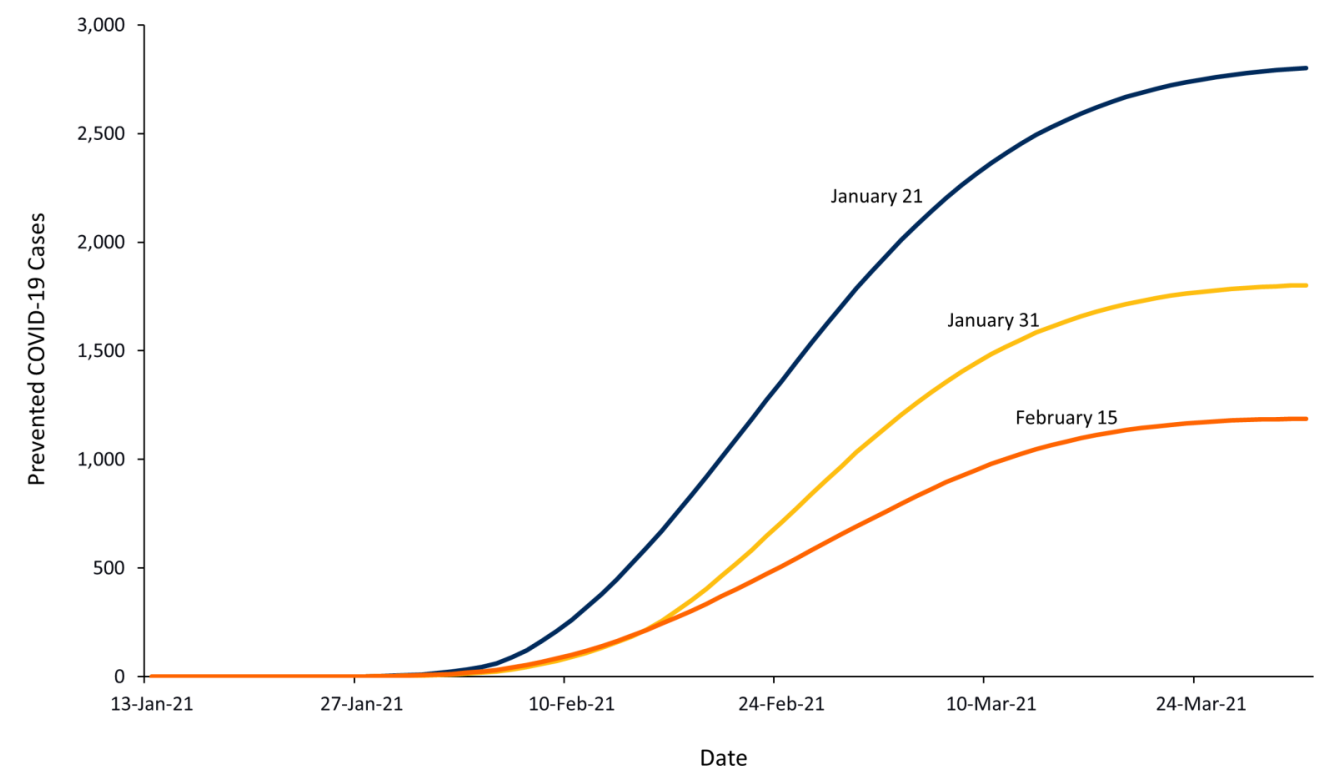

Figure 1. Projected Cumulative Number of Prevented COVID-19 Cases Among LTC Residents in Ontario According to Three Vaccine Rollout Scenarios

Projected cumulative number of prevented COVID-19 cases among Ontario LTC residents, from January 13 to March 31 , according to three vaccine rollout scenarios: vaccinating all Ontario LTC residents by January 21, vaccinating all Ontario LTC residents by January 31, and vaccinating all Ontario LTC residents by February 15, 2021. In all three scenarios, LTC residents in the Toronto, Peel, York, and Windsor-Essex regions are assumed to be vaccinated by January 21, 2021, in line with the province's current plan. The projected number of prevented COVID-19 cases is in comparison with a scenario of LTC residents receiving no vaccination.

If the province provided the first dose of the COVID-19 vaccine to all Ontario LTC residents by January 21, 2021, 2,800 cases of COVID-19 would be prevented (worst case, 665 ; best case, 4,900$)$.

If the province provided the first dose of the COVID-19 vaccine to all Ontario LTC residents by January 31, 2021, 1,800 cases of COVID-19 would be prevented (worst case, 600; best case, 3,000).

If the province provides the first dose of the COVID-19 vaccine to all Ontario LTC residents by February 15, 2021, as currently planned, 1,200 cases of COVID-19 would be prevented (worst case, 600; best case, 1,800).

Figure 2 presents the projected number of prevented COVID-19 deaths according to the three vaccine rollout scenarios. 


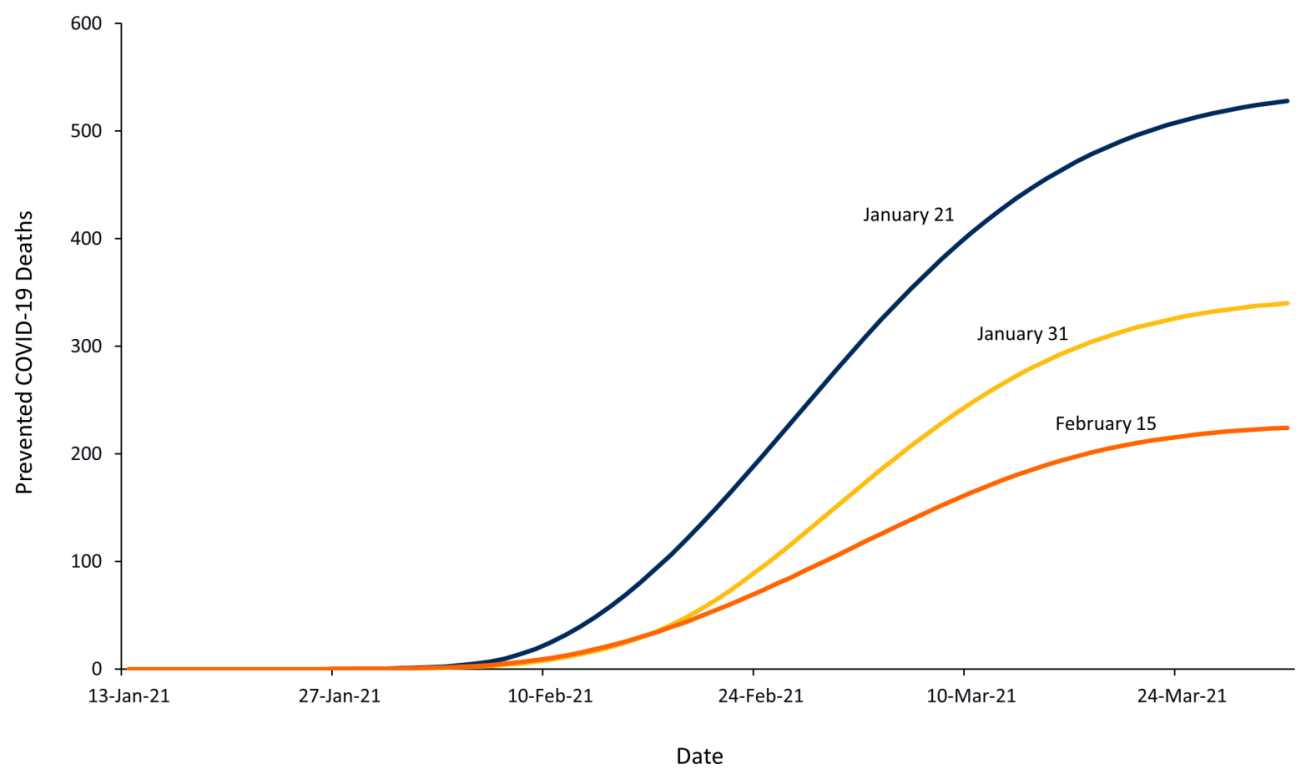

Figure 2. Projected Cumulative Number of Prevented COVID-19 Deaths Among LTC Residents in Ontario According to Three Vaccine Rollout Scenarios

Projected cumulative number of prevented COVID-19 deaths among Ontario LTC residents, from January 13 to March 31, according to three vaccine rollout scenarios: vaccinating all Ontario LTC residents by January 21, vaccinating all Ontario LTC residents by January 31, and vaccinating all Ontario LTC residents by February $15,2021$. In all three scenarios, LTC residents in the Toronto, Peel, York, and Windsor-Essex regions are assumed to be vaccinated by January 21, 2021, in line with the province's current plan. The projected number of prevented COVID19 deaths is in comparison with a scenario of LTC residents receiving no vaccination.

If the province provided the first dose of the COVID-19 vaccine to all Ontario LTC residents by January 21, 2021, 530 deaths of COVID-19 would be prevented (worst case, 120; best case, 900).

If the province provided the first dose of the COVID-19 vaccine to all Ontario LTC residents by January 31, 2021, 340 deaths of COVID-19 would be prevented (worst case, 100; best case, 580).

If the province provides the first dose of the COVID-19 vaccine to all Ontario LTC residents by February 15, 2021, as currently planned, 225 deaths of COVID-19 would be prevented (worst case, 100; best case, 340 ).

\section{Interpretation}

Accelerating the rollout of Ontario's COVID-19 vaccine such that all LTC residents receive the first dose of COVID-19 vaccine by January 31, 2021 would prevent a projected 600 COVID-19 cases and 115 deaths until March 31, 2021 when compared with the province's current plan to vaccinate all LTC residents by February 15, 2021. Further acceleration of the rollout would prevent even more COVID-19 cases and deaths.

If vaccine supply is limited, the early provision of first doses of a COVID-19 vaccine to LTC home residents is likely to be more beneficial than the on-schedule provision of second doses to health care workers outside of LTC homes. ${ }^{4}$ All LTC residents should receive the second dose according to approved vaccination schedules.

\section{Methods Used for This Science Brief}

We used a compartmental SIR model to forecast the number of COVID-19 cases and deaths between January 13 and March 31, 2021 among Ontario LTC residents, in a scenario of LTC residents receiving no vaccination. Figure 3 presents the three steps 
of the model. Step 1 predicts how many homes will have outbreaks based on the rates of community SARS-CoV-2 infection, step 2 predicts which LTC homes will experience a COVID-19 outbreak, and step 3 estimates the number of resident COVID-19 cases and deaths that will occur in each LTC home predicted to experience a COVID-19 outbreak.

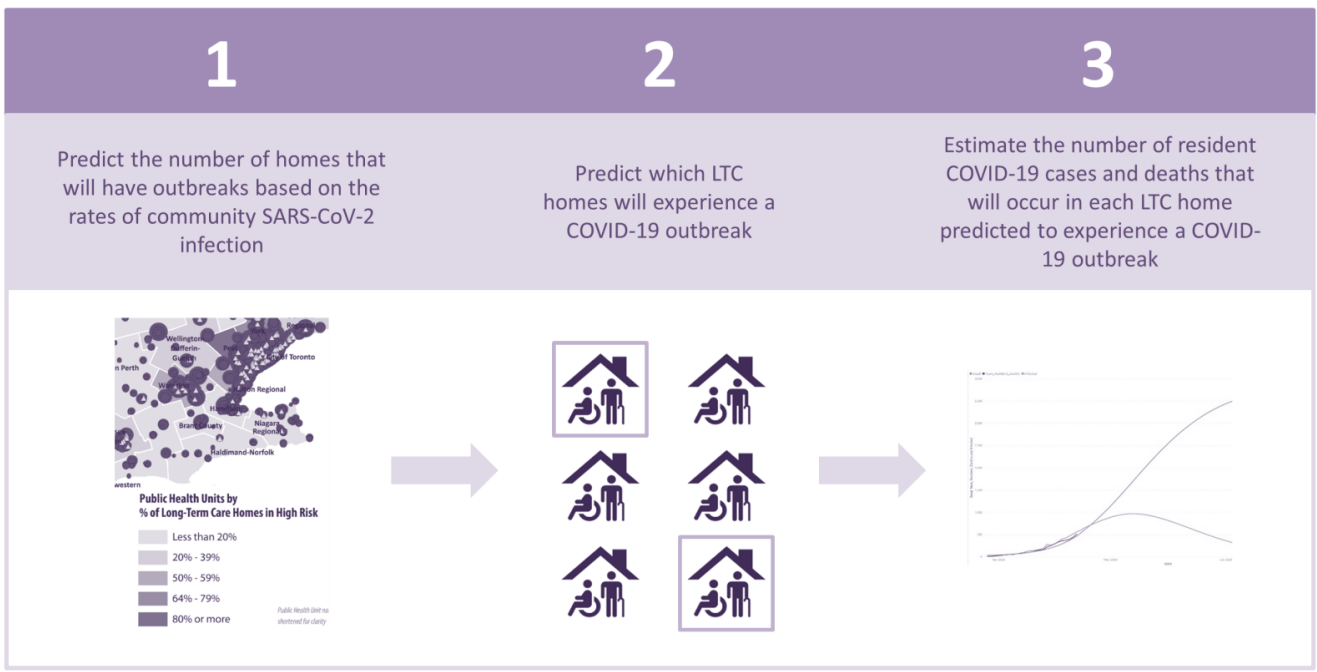

Figure 3. Approach to Modelling Ontario Long-Term Care Home COVID-19 Outbreaks

To forecast the number of COVID-19 cases and deaths among LTC residents in Ontario by March 31, 2021, under the three vaccine rollout scenarios, the compartmental SIR model also included the expected effect of COVID-19 vaccination, which was updated week-by-week based on LTC home specific effective reproduction numbers $\left(R_{e}\right),{ }^{1}$ that were modified in accordance with vaccine efficacy and incremental uptake among LTC residents.

We conservatively assumed a 90\% vaccine uptake among LTC residents, and a $85 \%$ efficacy for both Moderna's and Pfizer's COVID-19 vaccines, ${ }^{4}$ starting from day 14 after the first dose of the vaccine throughout the observation period, January 13 to March 31, 2021. We assumed that LTC residents received the second dose according to approved vaccination schedules.

We assumed that the SARS-CoV- 2 transmission in the community began with an $\mathrm{R}_{\mathrm{e}}$ of 1.08 on December 15, 2020, which decreased to 0.8 by January 31, 2021, using the prevalence of active cases on December 15, 2020, as starting point.

We specified worst-case and best-case scenarios. For the worst-case scenario, we assumed in step 1 an $R_{e}$ for a LTC home in outbreak of 1.2 on December 15, 2020, which decreased to 0.7 by early February 2021. In step 3, we assumed a basic reproduction number $\left(R_{0}\right)$ of 3.4 , which was modified daily using a logistic decay function for a period of 100 days.

For the best-case scenario, we assumed in step 1 an $R_{e}$ for a LTC home in outbreak of 1.08 , which decreased quickly to 0.7 on December 15, 2020, further decreasing to 0.4 by early February, 2021. In step 3 , we assumed an $R_{0}$ of 1.3 , which was again modified daily using a logistic decay function for a period of 100 days.

Additional assumptions included that the vaccine administration speed is constant, with a random order of homes being vaccinated, and that LTC residents previously infected with SARS-CoV-2 on or after September 1, 2020, have $100 \%$ durable immunity. In all three scenarios, LTC residents in the Toronto, Peel, York and Windsor-Essex regions were assumed to be vaccinated by January 21,2021 , in line with Ontario's current plan for vaccine rollout. 


\section{References}

1. Stall NM, Brown KA, Maltsev A, et al. COVID-19 and Ontario's long-term care homes. Sci Briefs Ont COVID-19 Sci Advis Table. 2021;2(7). https://doi.org/10.47326/ ocsat.2021.02.07.1.0

2. Government of Ontario. Ontario identifies key groups for distribution of initial COVID-19 vaccines. Ontario Newsroom. Published December 7, 2020. Accessed December 7, 2020. https://news.ontario.ca/en/release/59508/ontario-identifies-key -groups-for-distribution-of-initial-covid-19-vaccines

3. Government of Ontario. COVID-19 vaccination update. Technical Briefing presented at the: January 13, 2021; Ontario. Accessed January 19, 2020. https:// files.ontario.ca/moh-covid-19-vaccine-technical-briefing-en-january-13-2021-202101-13.pdf

4. Jüni $P$, Tuite AR, Bogoch II, et al. Rollout strategy for the Pfizer-BioNTech COVID19 vaccine in Ontario. Sci Briefs Ont COVID-19 Sci Advis Table. 2021;2(6). https:// doi.org/10.47326/ocsat.2021.02.06.1.0 